Case series

\title{
Nivolumab use for BRCA gene mutation carriers with recurrent epithelial ovarian cancer: A case series
}

\author{
Koji Matsuo $^{\mathrm{a}, \mathrm{b}, *, 1}$, Samantha E. Spragg, ${ }^{\mathrm{a}, 1}$, Marcia A. Ciccone ${ }^{\mathrm{a}}$, Erin A. Blake ${ }^{\mathrm{a}}$, Charité Ricker ${ }^{\mathrm{c}}$, \\ Huyen Q. Pham ${ }^{\mathrm{a}, \mathrm{b}}$, Lynda D. Roman ${ }^{\mathrm{a}, \mathrm{b}}$ \\ a Division of Gynecologic Oncology, Department of Obstetrics and Gynecology, University of Southern California, CA, USA \\ ${ }^{\mathrm{b}}$ Norris Comprehensive Cancer Center, University of Southern California, CA, USA \\ ${ }^{\mathrm{c}}$ Division of Medical Oncology, Department of Medicine, University of Southern California, CA, USA
}

\section{A R T I C L E I N F O}

\section{Keywords:}

Ovarian cancer

Recurrence

BRCA mutation carrier

Salvage therapy

Nivolumab

\begin{abstract}
A B S T R A C T
Tumors deficient in DNA mismatch repair are known to display increased susceptibility to immune checkpoint inhibitors due to accumulation of DNA damage and increased neoantigen load. This suggests that deficiency in the BRCA-related DNA repair mechanism may also be a surrogate marker for immunotherapy response. The aim of this study was to examine the efficacy of the immune checkpoint inhibitor, nivolumab, in women with $B R C A$ gene mutations and recurrent müllerian cancer. This retrospective case series followed six $B R C A$ carriers who received nivolumab monotherapy $(3.0 \mathrm{mg} / \mathrm{kg}$, intravenous, day 1 and 15 , every 4 weeks) as salvage therapy for recurrent epithelial ovarian $(n=5)$ and fallopian tubal $(n=1)$ cancers. Toxicity, treatment response, and survival were examined. Median age was 57 (range 51-64). BRCA1 and 2 mutations were equally distributed. All had high-grade serous histology, and all but one had advanced-stage disease at initial diagnosis. The majority had platinum-resistant disease $(n=4)$. All received salvage therapy prior to nivolumab therapy (median 3 lines), including PARP inhibitors $(n=3)$. The median number of nivolumab treatment cycles was 9, including 2 women receiving 18 cycles. Three women developed nivolumab-related toxicities, most commonly grade 2 hypothyroidism $(n=2)$. Median follow-up time was 13.4 months, and there were 3 complete responses, 1 partial response, and 2 patients with progressive disease. Objective response rate was $67 \%$ (4 out of 6 ). In conclusion, our study suggests that nivolumab monotherapy is well-tolerated and may be an effective salvage therapy for $B R C A$ mutation carriers with recurrent epithelial ovarian, fallopian tubal, and primary peritoneal cancers.
\end{abstract}

\section{Introduction}

In 2018, ovarian cancer remains the most deadly gynecologic malignancy in the United States (Siegel et al., 2018). The vast majority of patients ultimately recur, after which systemic chemotherapy plays a pivotal role. The currently available effective approaches for recurrent ovarian cancer include cytotoxic chemotherapy and biological targeting, such as anti-angiogenic and DNA repair inhibition. Recently, studies have shown that immunotherapy can be another effective treatment modality for recurrent disease.

In high-grade serous ovarian carcinoma, the most common histological type of ovarian cancer, nearly $15 \%$ of women harbor germline $B R C A$ mutations, and nearly half of tumors have alterations in the homologous recombination pathway (Liu and Konstantinopoulos, 2017). A recent study has shown that BRCA-mutated high-grade serous ovarian carcinomas have a high mutational load and more tumor-specific neoantigens (Strickland et al., 2016). In general, tumors with a high mutation burden have increased levels of neoantigens, which play a major role in the activity of immunotherapy (Hamanishi et al., 2016; Schumacher and Schreiber, 2015). These neoantigens recruit tumorinfiltrating $\mathrm{T}$ lymphocytes, resulting in a compensatory upregulation of the programmed cell death protein 1 (PD-1) pathway and suppression of the host immune system. PD-1 is a cell surface receptor that interacts with its ligand (PD-L1) to downregulate T cell activity (Ishida et al., 1992). Thus, targeting the PD-1 pathway with a checkpoint inhibitor is an attractive approach in hyper-mutated tumors.

A recent clinical trial has shown anti-tumor activity with immune checkpoint inhibitors against tumors with mismatch repair deficiencies (Le et al., 2017; 2015). This proof-of-principal study led to the theory that inhibiting the PD-1/PDL-1 pathway may be effective in sustaining

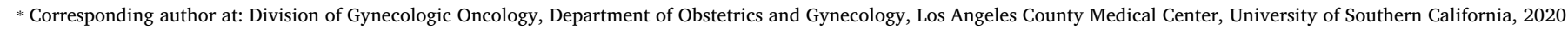
Zonal Avenue, IRD520, Los Angeles, CA 90089, USA.

E-mail address: koji.matsuo@med.usc.edu (K. Matsuo).

${ }^{1}$ Contributed equally to the work. 
BRCA mutation $\rightarrow$ high burden of mutations

I

High tumor mutation burden $\rightarrow$ more tumor surface neoantigens

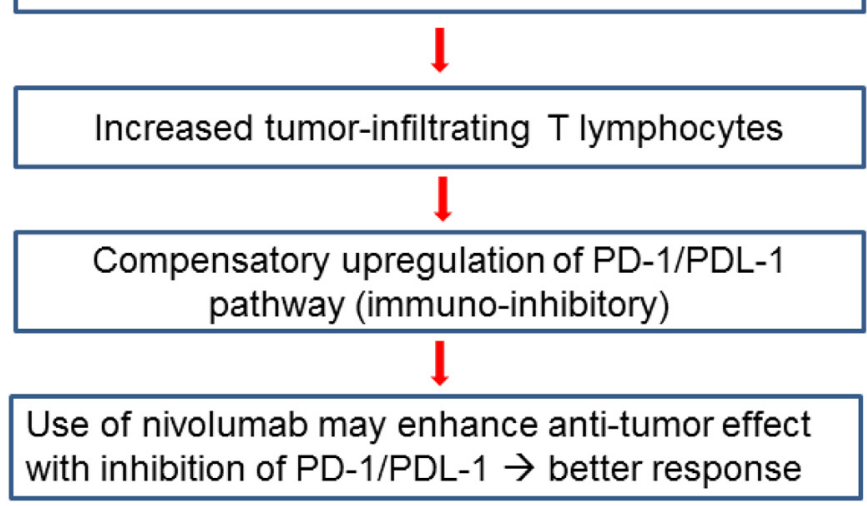

Fig. 1. Schema for proposed rationale of the study.

T lymphocyte activity against BRCA-mutated tumors.

Based on this mechanism (Fig. 1), we hypothesized that the immune checkpoint inhibitor, nivolumab, may be effective against recurrent epithelial ovarian cancer in women harboring a BRCA gene mutation. The objective of the study was to describe the effectiveness of an immune checkpoint inhibitor, nivolumab, on patients with recurrent ovarian cancer and a BRCA mutation in a retrospective case series.

\section{Patients and methods}

\subsection{Eligibility criteria}

Upon obtaining Institutional Review Board approval at the University of Southern California (USC), an institutional database was utilized to retrospectively identify eligible cases between January 2016 and December 2017 at Los Angeles County USC Medical Center and USC Keck Medical Center. The inclusion criteria were women with germline BRCA 1/2 mutations and recurrent epithelial ovarian, fallopian tubal, or primary peritoneal carcinoma who received nivolumab as salvage therapy. Patient demographics, tumor characteristics, treatment and response details, adverse events, and survival outcomes were abstracted from medical records.

\subsection{Clinical information}

Patient demographics included age at nivolumab treatment, race/ ethnicity, and BRCA mutation type. Tumor characteristics at initial diagnosis included cancer type, stage, histology, and platinum sensitivity status. Treatment history included previous utilization of poly ADP ribose polymerase (PARP) inhibitors, lines of salvage chemotherapy prior to nivolumab, and the details of nivolumab therapy (dose, schedule, and number of cycles administered). Treatment response included objective response rate and clinical benefit rate. Adverse events during nivolumab therapy were also collected. Survival outcomes included progression-free survival and overall survival.

\subsection{Study definition}

Cancer stage was based on the 2014 International Federation of Gynecology and Obstetrics (FIGO) criteria. Treatment response was assessed via the immune-related Response Evaluation Criteria in Solid Tumor (iRECIST, version 1.0) (Seymour et al., 2017). Objective response rate was defined as either complete response or partial response. Clinical benefit rate was defined as complete/partial responses and stable disease. Treatment-related toxicity was assessed with the National Cancer Institute's Common Terminology Criteria for Adverse Event (CTC-AE, version 5.0). Progression-free survival was defined as the time interval between the initiation of nivolumab and the first progression of disease or death. Overall survival was defined as the time interval between the initiation of nivolumab and death from disease any reason (all-cause). Women with the above survival events were censored at the last follow-up.

\subsection{Statistical consideration}

Continuous variables were assessed for normality, expressed with mean and standard deviation or median and range as appropriate. Categorical variables were expressed with number and percent proportion. Standard descriptive analysis was performed for this case series.

\section{Results}

Six women met the inclusion criteria and their demographics are summarized in Table 1 . The median age at initiation of nivolumab treatment was 57 years (range 51-64), and the majority were younger than 60 years of age $(n=4,67 \%)$. Race/ethnicity varied (Caucasian, non-Hispanic $n=2,33 \%$, Asian $n=2,33 \%$, and Hispanic $n=1,17 \%$ ). $B R C A$ mutations were equally divided between BRCA1 $(n=3,50 \%)$ and BRCA2 $(n=3,50 \%)$.

Tumor characteristics are shown in Table 2. High-grade serous ovarian carcinoma constituted the majority of cases $(n=5,83 \%)$. All but one woman had advanced-stage disease at diagnosis (stage III-IV $n=5,83 \%$ ). All women received a platinum and taxane doublet as initial therapy. Prior to nivolumab initiation, all women received salvage chemotherapy: 4 (67\%) women received 3 or fewer regimens, and $2(33 \%)$ women received $>6$ regimens. The majority of the study population had platinum resistant disease $(n=4,67 \%)$. Three $(50 \%)$ women were previously treated with a PARP inhibitor.

Treatment type is shown in Table 3. Treatment dose for nivolumab was $3.0 \mathrm{mg} / \mathrm{kg}$, intravenously, day 1 and 15 , every four weeks in all patients. A median of 9 (range 3-18) cycles were administered. Three (50\%) women received $>10$ cycles. Three $(50 \%)$ women achieved a complete response, one (17\%) had a partial response, and two (33\%) experienced progressive disease. These statistics indicate that both objective response rate and clinical benefit rate were $67 \%$.

Toxicity profile related to nivolumab therapy is shown in Table 3. There were three $(50 \%)$ women who developed toxicity related to nivolumab (all were grade 2). The most common toxicity was hypothyroidism (median time to onset, 3 months), all cases of which were successfully treated with oral levothyroxine. One patient (Case 3) opted to hold nivolumab treatment due to thyroid lab abnormalities. Despite cessation, the treatment response has been maintained after nine months off the drug. In another patient (Case 2) nivolumab treatment was held due to developing lichen planus after 18 cycles, and she

Table 1

Patient demographics.

\begin{tabular}{llllll}
\hline No. & Age & Race/Ethnicity & Cancer type & Gene & Mutation \\
\hline 1 & 64 & Asian & PPC & BRCA2 & c.3744_3747delTGAG \\
2 & 63 & Other & HGSOC & BRCA1 & c.5444G > A \\
3 & 52 & Caucasian & HGSOC & BRCA1 & Deletion exons 1-7 \\
4 & 59 & Caucasian & HGSOC & BRCA2 & c.5946delT \\
5 & 55 & Asian & HGSOC & BRCA2 & c.276dupA \\
6 & 51 & Hispanic & HGSOC & BRCA1 & c.4327C > T
\end{tabular}

Abbreviations: HGSOC, high-grade serous ovarian carcinoma; and PPC, primary peritoneal carcinoma 
Table 2

Tumor characteristics and prior treatment type.

\begin{tabular}{lllll}
\hline No. & Cancer stage & $\begin{array}{l}\text { Platinum } \\
\text { sensitivity }\end{array}$ & $\begin{array}{l}\text { Prior salvage } \\
\text { chemo }^{\text {a }}\end{array}$ & $\begin{array}{l}\text { PARP inhibitor } \\
\text { use }\end{array}$ \\
\hline 1 & IIIC & Sensitive & 2 & No \\
2 & IC & Resistant & 2 & No \\
3 & IIIC & Sensitive & 2 & No \\
4 & IV & Resistant & $>6$ & Yes \\
5 & IIIB & Resistant & $>6$ & Yes \\
6 & IIIC & Resistant & 3 & Yes \\
\hline
\end{tabular}

a number of regimens prior to the nivolumab therapy is shown.

Table 3

Treatment response, toxicity, and outcome.

\begin{tabular}{lllllll}
\hline PT & Cycle $^{\mathrm{a}}$ & Response & Current status & Toxicities & PFS & OS \\
\hline 1 & 18 & PR & On treatment & None & - & 17 \\
2 & 18 & CR & Treatment on hold & Lichen planus (G2) & - & 18.4 \\
3 & 5 & CR & Treatment on hold & Hypothyroidism (G2) & - & 9.7 \\
4 & 11 & PD & DOD & None & 11 & 14 \\
5 & 3 & PD & DOD & None & 5.5 & 5.5 \\
6 & 7 & CR & On treatment & Hypothyroidism (G2) & - & 5.7 \\
\hline
\end{tabular}

Abbreviations: DOD, die of disease; PFS, progression-free survival; and OS, overall survival.

a nivolumab therapy.

b due to lichen planus.

c due to patient request.

remains without disease 4 months following her last treatment. One woman (Case 4) received nivolumab with venetoclax and decitabine in the setting of pre-existing myelodysplastic syndrome. This patient had received PARP inhibitor therapy previously.

The median follow-up time of the censored cases after nivolumab initiation was 13.4 months (range, 5.7-18.4). There were two (33\%) women who experienced disease progression and died of disease, and the progression-free times of the two patients were 5.5 and 11 months, respectively. Patients whose disease progressed through nivolumab therapy received more than six chemotherapy regimens prior to nivolumab, including a PARP inhibitor, whereas those who responded to nivolumab therapy received fewer cycles of chemotherapy prior to nivolumab initiation ( 2 cycles $n=4$, and 3 cycles $n=1$; Tables 1 and 3 ).

\section{Discussion}

Treatment outcome observed in our study partly supports the recent clinical trial examining the effectiveness of another PD-1/PD-L1 inhibitor, pembrolizumab, against solid tumors with mismatch repair deficiency (Le et al., 2015). In their study, five (71\%) out of seven patients with non-colorectal cancer whose tumors had a deficiency in the mismatch repair mechanism had an objective response to pembrolizumab. Of note, none ( 0 out of 18 ) responded to pembrolizumab when the tumors were mismatch repair proficient.

Thus, the objective response rate of $67 \%$ in our study (four out of six) seems comparable to that of pembrolizumab treatment for mismatch repair deficient tumors. Therefore, it is biologically plausible that the increased tumor mutation burden associated with BRCA mutations results in increased tumor surface neoantigens which trigger the host immune response, and nivolumab, used to inhibit the compensatory upregulation of the PD-1/PD-L1 pathway, is effective for this reason (Fig. 1).

In this study, lack of a control group limits the interpretation of our results. The current study only examined $B R C A$ mutation carriers and did not include those with wild-type $B R C A$ status. In a review of the literature, there is one clinical trial with a phase IIa study design that examined the safety and effectiveness of nivolumab in women with recurrent platinum-resistant ovarian cancer (Hamanishi et al., 2015).
Nearly half of the women included were heavily pre-treated and had received four or more regimens prior to the nivolumab therapy (55\%). Notably, an objective response was seen in three $(15 \%)$ out of twenty patients including two (10\%) patients with a durable complete response. Of note, this study did not have information for germline $B R C A$ status.

Their findings regarding the efficacy of nivolumab therapy seem comparable to those reported in recent clinical trials for platinum-resistant disease (pooled objective response rate 16\% including $10 \%$ for monotherapy and 22\% for combination therapy) (Matsuo et al., 2010). While intriguing to speculate that nivolumab could be particularly effective in BRCA mutation carriers, this association needs to be further examined in prospective studies.

In our study, the two women who had disease progression with nivolumab had received more than six previous cycles of treatment. This finding implies the following factors to consider when administering nivolumab: decreased bone marrow reserve and increased tumor burden. In general, bone marrow reserve is expected to be decreased after multiple lines of chemotherapy. In this case, regimens that block the PD-1/PD-L1 pathway may not be effective due to insufficient immune-effectors. In addition, tumor burden may be more significant after multiple lines of chemotherapy failure. A recent retrospective study found that large tumor size is an independent predictor for checkpoint inhibitor failure (Boland et al., 2018). Thus, if a checkpoint inhibitor is considered in women with recurrent ovarian cancer, earlier use when tumor burden is small would be the key to avoiding treatment failure. In this setting, universal multi-gene panel testing for women with newly diagnosed epithelial ovarian cancer would be integral to early identification of the patients with germline BRCA mutations (NCCN Guidelines).

Clinical utility of our observations may extend to tumors harboring homologous recombination deficiencies. Tumors with wild-type BRCA but homologous mutations also have a higher mutation burden resulting in a higher tumor surface neoantigen load compared to wildtype BRCA and homologous recombination proficient tumors (Strickland et al., 2016). Compensatory upregulation of the PD-1/PD-L1 pathway may also occur in this setting thus possibly making a checkpoint inhibitor effective.

\section{Conclusion}

Our case series suggests that salvage treatment with nivolumab monotherapy is well tolerated and may achieve prolonged benefit in women with recurrent epithelial ovarian cancer with a BRCA mutation.

\section{Disclosure statement}

The authors declare no conflict of interest.

\section{Author contributions}

Conceptualization, L.D.R, K.M.; data curation, all; formal analysis, K.M.; funding acquisition, K.M., L.D.R.; methodology, K.M.; writing original draft, K.M., S.E.S.; editing and reviewing, all authors.

\section{Funding support}

Ensign Endowment for Gynecologic Cancer Research (K.M.).

\section{References}

Boland, J., Martin, M., Zecca, N., Iasonos, A., Zhou, Q., Aghajanian, C.A., Sabbatini, P., Cadoo, K.A., Zamarin, D., 2018. Predictors of Early Treatment Discontinuation in Ovarian Cancer Patients on Checkpoint Blockade Immunotherapy. 49th Annual Meeting on Women's Cancer, New Orleans, LA. (March 24-27, 2018).

Hamanishi, J., Mandai, M., Ikeda, T., Minami, M., Kawaguchi, A., Murayama, T., Kanai, M., Mori, Y., Matsumoto, S., Chikuma, S., et al., 2015. Safety and antitumor activity 
of anti-PD-1 antibody, Nivolumab, in patients with platinum-resistant ovarian cancer. J. Clin. Oncol. 33, 4015-4022.

Hamanishi, J., Mandai, M., Matsumura, N., Abiko, K., Baba, T., Konishi, I., 2016. PD-1/ PD-L1 blockade in cancer treatment: perspectives and issues. Int. J. Clin. Oncol. 21, $462-473$.

Ishida, Y., Agata, Y., Shibahara, K., Honjo, T., 1992. Induced expression of PD-1, a novel member of the immunoglobulin gene superfamily, upon programmed cell death. EMBO J. 11, 3887-3895.

Le, D.T., Uram, J.N., Wang, H., Bartlett, B.R., Kemberling, H., Eyring, A.D., Skora, A.D., Luber, B.S., Azad, N.S., Laheru, D., et al., 2015. PD-1 blockade in tumors with mismatch-repair deficiency. N. Engl. J. Med. 372, 2509-2520.

Le, D.T., Durham, J.N., Smith, K.N., Wang, H., Bartlett, B.R., Aulakh, L.K., Lu, S., Kemberling, H., Wilt, C., Luber, B.S., et al., 2017. Mismatch repair deficiency predicts response of solid tumors to PD-1 blockade. Science 357, 409-413.

Liu, J.F., Konstantinopoulos, P.A., 2017. Chapter 6 - homologous recombination and BRCA genes in ovarian Cancer: clinical perspective of novel therapeutics. Part I: ovarian cancer. In: Translational Advances in Gynecologic Cancers. 2017. pp. 111-128.

Matsuo, K., Lin, Y.G., Roman, L.D., Sood, A.K., 2010. Overcoming platinum resistance in ovarian carcinoma. Expert Opin. Investig. Drugs 19, 1339-1354.

Schumacher, T.N., Schreiber, R.D., 2015. Neoantigens in cancer immunotherapy. Science $348,69-74$.

Seymour, L., Bogaerts, J., Perrone, A., Ford, R., Schwartz, L.H., Mandrekar, S., Lin, N.U., Litiere, S., Dancey, J., Chen, A., et al., 2017. iRECIST: guidelines for response criteria for use in trials testing immunotherapeutics. Lancet Oncol. 18, e143-e152.

Siegel, R.L., Miller, K.D., Jemal, A., 2018. Cancer statistics, 2018. CA Cancer J. Clin. 68 7-30.

Strickland, K.C., Howitt, B.E., Shukla, S.A., Rodig, S., Ritterhouse, L.L., Liu, J.F., Garber J.E., Chowdhury, D., Wu, C.J., D'Andrea, A.D., et al., 2016. Association and prognostic significance of BRCA1/2-mutation status with neoantigen load, number of tumor-infiltrating lymphocytes and expression of PD-1/PD-L1 in high grade serous ovarian cancer. Oncotarget 7, 13587-13598. 